\section{Muons as Probes in Solids}

\author{
W. Kündig and P.F. Meier, Zurich
}

(Physik-Institut der Universität Zürich)

If spin polarized muons are stopped in a solid, the muon spin will precess at the Larmor frequency in the local magnetic field until the muon decays (the lifetime is $2.2 \mu \mathrm{s})$. By detecting the time dependent angular correlation pattern of the decay positron, the muon spin rotation ( $\mu \mathrm{SR}$ ) can be observed and thus local magnetic fields can be measured.

Negatively charged muons $\left(\mu^{-}\right)$behave in matter like heavy electrons. They are attracted by the electric fields of the nuclei and fall rapidly into their atomic ground state which has a radius about two hundred times smaller than that of the lowest electron states. Positively charged muons $\left(\mu^{-}\right)$on the other hand, behave in condensed matter like light unstable protons. They are repelled by nuclei and go preferentially to interstitial regions. In metals their charge is screened by conduction electrons, whereas in semiconductors and insulators a $\mu^{+}$ may capture an electron to form a paramagnetic state called muonium.

\section{History}

Within two days in January 1957 three manuscripts were submitted to Physical Review, each one describing a separate novel experiment confirming the violation of parity in weak interactions, as suggested by Lee and Yang. The first (Wu et al.) reported on the experiments on $\beta^{-}$decay of polarized nuclei. In the second Garwin, Lederman and Weinrich, by inventing the muon spin rotation technique, showed that parity is violated in both the

and the

$$
\pi^{+} \rightarrow \mu^{+} v_{\mu}
$$

$\mu^{+} \rightarrow \mathrm{e}^{+}+v_{\mathrm{e}}+\bar{v}_{\mu}$

decays. The third paper by Friedman and Telegdi introduced the notion of muonium and noted the spin-depolarizing effects arising from its hyperfine interaction and the rapid precession of its total magnetic moment in an external magnetic field. These last two papers set the stage for $\mu$ SR and established the principles on which most applications in solid-state physics are based.

It is remarkable that in the paper by Garwin et al. appeared already the speculation "... it seems possible that polarized positive and negative muons will be- come a powerful tool for exploring magnetic fields in nuclei, atoms and interatomic regions". Applications to solid state physics were, however, rare until the construction of the meson factories about ten years ago. At present $\mu \mathrm{SR}$ is done at SIN (Villigen, Switzerland), TRIUMF (Vancouver), LAMPF (Los Alamos), CERN, DUBNA, KEK (Japan), and in the near future in Brookhaven, at NIKHEF (Amsterdam) and at SNS, the spallation neutron source at the Rutherford-Appleton Laboratory in England. Although the method is limited by the few existing muon beams, $\mu$ SR activity has increased dramatically. The progress and status of $\mu \mathrm{SR}$ research can best be found by consulting the proceedings of the three $\mu \mathrm{SR}$ conferences given in Reference 1.

\section{$\mu$ SR Technique}

To the best of present day knowledge, the muon is nothing more than a heavy electron. Some of its properties are

mass $\quad m_{\mu}=105.659 \mathrm{MeV} / \mathrm{c}^{2}$ spin $\quad=0.112610 \mathrm{M}_{\mathrm{p}}$ lifetime $\quad \tau_{\mu}=2.19709 \mu \mathrm{s}$ magnetic moment $\mu_{\mu}=3.18335 \mu_{\mathrm{p}}$ gyromagn. ratio $\quad \gamma_{\mu} / 2 \pi=135.537 \mathrm{MHz} / \mathrm{T}$

Fig. 1 shows in the upper part the pion decay (1) and the muon decay (2) at maximum possible energy. When the muon is produced it is fully longitudinally polarized in the pion rest-frame. When it decays it follows from conservation laws that the positron is emitted in the muon spin direction. The angular correlation pattern for this case is as shown on the left of the figure. If all the positrons in the decay are considered the angular correlation function is:

$\left[1+A P(t) \cos \left(\theta+\omega_{L} t\right)\right], \quad$ (3)

where $\theta$ is the angle between the muon spin and the direction of the decay positron, giving the angular correlation pattern shown on the right. For negative muons the cosine term is negative. If an external magnetic field is applied perpendicular to the incoming muon spin, direction, the spin will precess with the Larmor frequency $\omega_{L}=\gamma_{\mu} B$.

The three quantities obtained from a $\mu \mathrm{SR}$ experiment are:
$N(\theta, t)=N_{0} \exp \left(-t / \tau_{\mu}\right) \times$

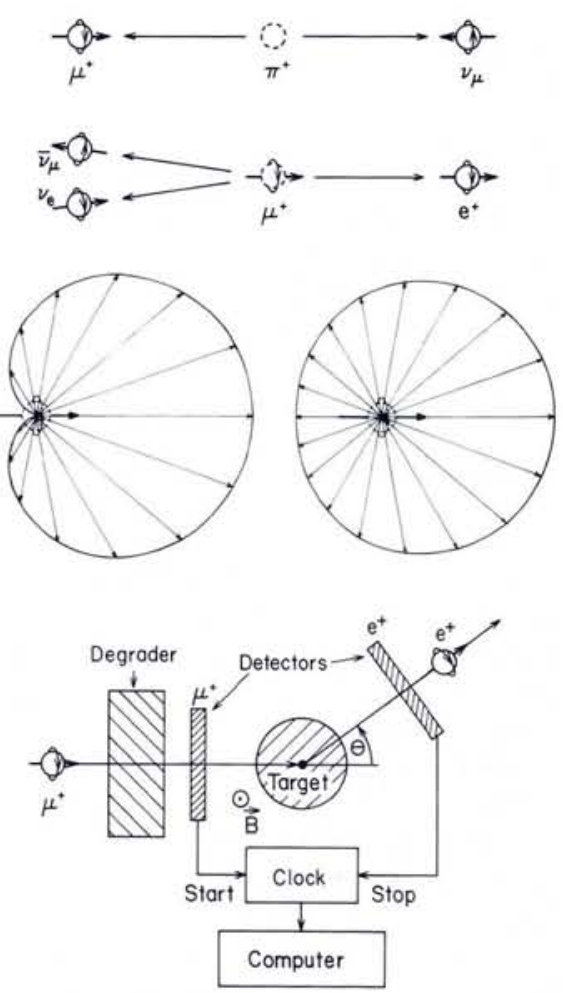

Fig. 1 - The upper part of the figure illustrates the $\pi^{+}$and $\mu^{+}$decay. In the centre, the angular distribution patterns of the decay positrons with respect to the $\mu^{+}$spin are shown when only positrons at the maximum energy are registered (left), and all the positrons are detected (right). In the lower part is a simplified diagram of a $\mu S R$ apparatus.

1) the anisotropy $A$ : if all muons are in the same state, $A=1 / 3$.

2) the Larmor frequency $\omega_{L}$ : this is a direct measure of the field $B_{\mu}$ at the muon.

3) the relaxation function $P(t)$ : in a transverse field this measures field inhomogeneities e.g. due to nuclear dipolar fields. The form of $P(t)$ lexponential or Gaussian decay) gives information about the motion of the muons.

As an example, Fig. 2 shows the $\mu \mathrm{SR}$ spectrum of polycrystalline copper measured at room temperature in an external transverse field of $50.6 \mathrm{mT}$. The observed frequency $\omega_{\mathrm{L}}=\gamma B$, the amplitude after corrections for solid angle effects $A=1 / 3$; no relaxation is observed i.e. $P(t)=1$.

\section{$\mu \mathrm{SR}$ in Ferromagnetic Cobalt}

$\mu \mathrm{SR}$ can be used to measure directly local fields at interstitial sites in ferroand antiferromagnets. One of the first series of measurements made at SIN will serve as an example. These were on an ellipsoidal single crystal of cobalt in zero external field over a range of temperatures. The results shown in Fig. 3 were quite unexpected. The local field $B_{\mu}$ 


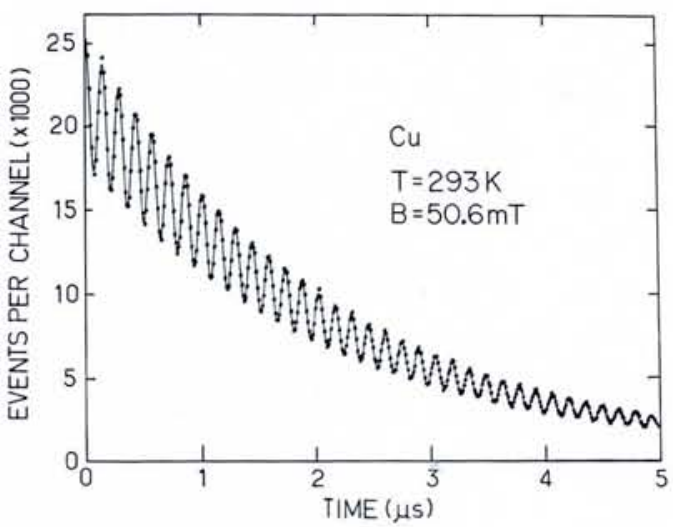

Fig. $2-\mu S R$ spectrum of $\mathrm{Cu}$ in a transverse field of $50.6 \mathrm{mT}$. The oscillations superimposed on the exponential muon decay are due to the muon spin precession. The line plotted corresponds to the least square fit to Eq. (3).

changes from $-31.7 \mathrm{mT}$ at $\mathrm{OK}$ to +40 $\mathrm{mT}$ at $690 \mathrm{~K}$. At the HCP-FCC phase transition at $690 \mathrm{~K}, B_{\mu}$ jumps to $+74 \mathrm{mT}$ and then follows approximately the magnetization curve.

In a non-magnetized sample the local field is:

$$
\mathbf{H}_{\mu}=\mathbf{B}_{\mu} / \mu_{\mathrm{o}}=\mathbf{H}_{\mathrm{L}}+\mathbf{H}_{\text {dip }}+\mathbf{H}_{\mathrm{hf}} \text { (4) }
$$
where $H_{L}=1 / 3 M_{s}$ is the Lorentz field which is proportional to the saturation magnetization, and $H_{\text {dip }}$ is the dipolar field from the atomic dipoles inside the Lorentz sphere. The hyperfine field $H_{\text {hf }}$ (= Fermi contact field) measures directly the local spin density at the interstitial muon site $\left(H_{\mathrm{hf}}=-4 / 3 \mu_{B} \rho_{\text {spin }}\right)$. So $\mu \mathrm{SR}$ offers the unique possibility of measuring the spin density at interstitial sites, and thus complements neutron diffraction experiments, which are insensitive to interstitial spin densities.

The dipolar field depends on the site of the muon and the direction and magnitude of the local Co moments. The direction of the magnetization is known to change from being parallel to the hexagonal axis for $T<520 \mathrm{~K}$ to perpendicular for $T>600 \mathrm{~K}$. This in turn changes the dipolar field at the muon by a factor of two and is reflected in the observed rapid change of the Larmor frequency in this temperature region. Three sites in the crystal lattice appear as reasonable stopping sites for a muon, the tetrahedral and octahedral interstitial sites, and the substitutional site, if the muon is trapped by a vacancy. The calculated fields $B_{\text {dip }}$ (tet) and $B_{\text {dip }}$ (oct) are shown as dashed and solid lines respectively in Fig. 3. A comparison with the measured value indicates that $B_{\mu}$ follows roughly $B_{\text {dip }}$ (oct). This measurement as well as those made in external fields show unambiguously that the muon in $\mathrm{Co}$ is at the octahedral site or diffuses between the octahedral sites. The results in Co, discussed in more detail in a review arti$\mathrm{cle}^{2}$ ) are for $T=0 \mathrm{~K}$ :

$$
\begin{aligned}
& B_{\mu}=-31.7 \pm 1.0 \mathrm{mT} \\
& B_{\text {hf }}=-610 \pm 20 \mathrm{mT} \\
& B_{\text {dip }}=-24.2 \mathrm{mT} \\
& \rho_{\text {spin }}=0.039 \mu_{B} / \AA^{3}
\end{aligned}
$$

The muon is at the octahedral interstitial site.

Similar results have been obtained for other elemental ferromagnets as well as for some antiferromagnetic compounds.

\section{Muon Diffusion in Metals}

In ferromagnetic iron, which has a bcc structure, both the octahedral and tetrahedral interstitial sites have tetragonal symmetry. In the unit cell there are thus several sites which are chemically equivalent but, for a general direction of magnetization, magnetically different. Stationary muons would occupy randomly the chemically equivalent sites and one would expect different Larmor precession frequencies depending on the different dipolar fields. Over a large temperature interval one observes, however, a single frequency which indicates that the muon diffuses rapidly in Fe so that the different field contributions are averaged out. At very low temperatures, the relaxation rate of the signal increases indicating a slowing down of the diffusion.

In non-magnetic metals the muon precession is in general disturbed by dipolar fields which are produced by the magne-
Fig. 3 - Temperature dependence of the measured local field $B_{u}$ and the contributions to $B_{u}$ in Co. The calculated dipolar field $B_{\text {dip }}$ is given for the octahedral site (solid line) and the tetrahedral site $B_{\text {dip }}$ is due to a change in the easy axis of magnetization. At $690 \mathrm{~K}$ Co changes from hcp to fcc. A comparison of $B_{\text {dip }}$ and $B_{\mu}$ shows that the muon must be at the octahedral site. (dashed line). The sign change in tic moments of neighbouring nuclei. These nuclear spins are not ordered and thus produce at the interstitial sites variable dipolar fields of the order of $10^{-4}$ T. The Larmor precession of stationary muons in a transverse magnetic field of about $0.1 \mathrm{~T}$ is slightly disturbed by these additional randomly oriented dipolar fields and muons at sites with different fields precess at different frequencies. This results in an effective relaxation function given by:

$$
P(t)=\exp \left(-\sigma^{2} t^{2}\right)
$$

The quantity $\sigma^{2}$ is a measure of the width of the dipolar field distribution and depends on the site of the muon, its distance to the neighbouring nuclei, as well as on the orientation of the external field with respect to the crystal axes. This enabled the determination of the muon site in $\mathrm{Cu}$. It was found that the $\mu^{+}$is stationary at the octahedral interstitial sites below $80 \mathrm{~K}$ and that the positions of the nearest $\mathrm{Cu}$ neighbors are slightly distorted. This lattice relaxation around the impurity lowers the energy. The muon is self-trapped and forms a small polaron. The non-vanishing electric field gradient at the nearby $\mathrm{Cu}$ nuclei has also been measured by observing the influence of the quadrupolar interaction of the $\mathrm{Cu}$ spins on the muon precession at lower external field strengths.

At temperatures above $80 \mathrm{~K}$ the muons start to diffuse in the Cu lattice, thereby averaging out the different dipolar field contributions which results in a reduction of the relaxation rate and in the change of the form of the relaxation function to an exponential:

$$
P(t)=\exp (-\lambda t)
$$

In Fig. 4, the change of the relaxation rate in $\mathrm{Cu}$ as a function of temperature is shown. Above room temperature the diffusion is already so fast that within the

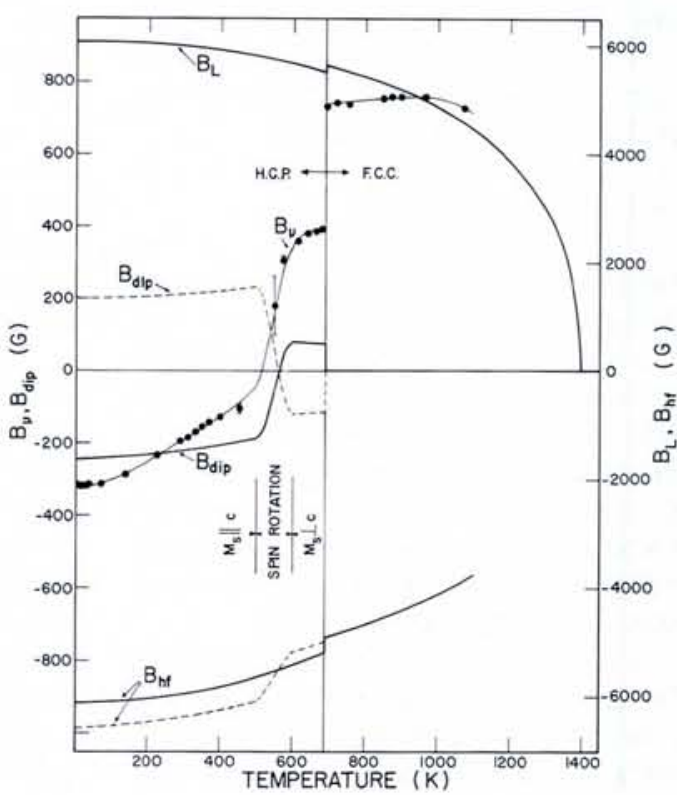




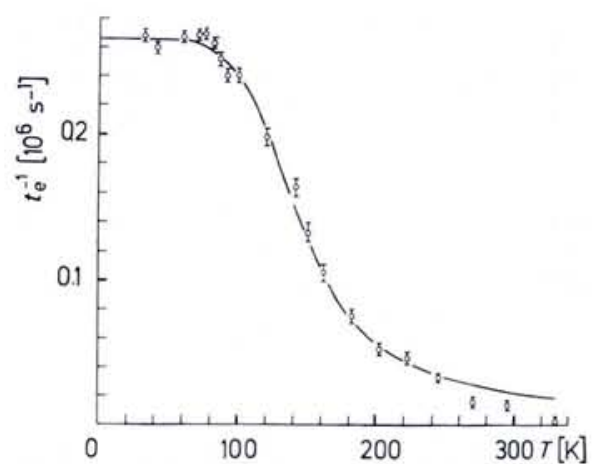

experimental limits due to the finite muon lifetime, no depolarization is observed.

The change-over from the stationary relaxation function (5) to the one that has been averaged by motion (6) is usually described by

$P(t)=$

$\exp \left\{-2 \sigma^{2} \tau_{\mathrm{c}}^{2}\left[\exp \left(-t / \tau_{\mathrm{c}}\right)-1+t / \tau_{\mathrm{c}}\right]\right\}$

The correlation time $\tau_{c}$ is directly related to the diffusion coefficient $D$. A fit of the time spectra to this relaxation function then yields $D$ as a function of $T$. In $\mathrm{Cu}$, where $D=a^{2}{ }_{o} / 12 \tau_{c}$, it was found that $D$ follows essentially an Arrhenius type temperature behaviour $D=4.4 \times 10^{-13}$ $\exp [-48 \mathrm{meV} / \mathrm{kT}] \mathrm{m}^{2} \mathrm{~s}^{-1}$. The activity energy of $48 \mathrm{meV}$ is very low compared to the $400 \mathrm{meV}$ measured for hydrogen diffusion in $\mathrm{Cu}$ but can be explained by phonon-assisted tunnelling between ground states for which the isotope effect is rather large.

In recent experiments, a decrease of the relaxation rate in $\mathrm{Cu}$ below $20 \mathrm{~K}$ was observed. This is an indication for the onset of coherent diffusion which also occurs for muons in other metals. In pure Al samples, e.g., no depolarization is observed down to $30 \mathrm{mK}$. Information about the diffusion can, however, be obtained indirectly from measurements on samples which are electron-irradiated or doped with impurities. Coherent diffusion is very sensitive to deviations from the periodic lattice potential produced by the strain fields. It is found that muons are trapped near vacancies or impurities but are released at elevated temperatures. The dependence of the relaxation rate on the impurity content of the sample then allows one to determine the diffusivity in the pure sample. These $\mu \mathrm{SR}$ experiments have provoked a renewed interest in the theory of quantum diffusion of light particles but many problems are still not understood.

$\mu \mathrm{SR}$ in Semiconductors and Insulators

If a positive muon is stopped in an insulator or semiconductor, it may capture an electron and form muonium $\left(\mathrm{Mu}=\left(\mu^{+} \mathrm{e}^{-}\right)\right)$. Since the muon has a large mass compared to the electron
Fig. 4 - Reciprocal of time $t_{\mathrm{e}}$ in which the precession signal in Cu decays by a factor $e^{-1}\left[P\left(t_{e}\right)=1 / e\right]$. The solid line corresponds to the least square fit to $E q$. (7) where $\tau$ follows an Arrhenius type behaviour.

(the reduced electron mass is $m_{\mathrm{e}}^{*}=$ $\left.m_{\mathrm{e}} /\left(1+m_{\mathrm{e}} / m_{\mu}\right)=0.9952 m_{\mathrm{e}}\right)$ many properties of the $\mathrm{Mu}$ and $\mathrm{H}$ atom are similar. E.g. the binding energies and the $1 \mathrm{~s}$ radii are

$$
\begin{array}{lr}
E_{\mathrm{H}}=13.598 \mathrm{eV} & a_{\mathrm{H}}=0.5295 \AA \\
E_{\mathrm{Mu}}=13.540 \mathrm{eV} & a_{\mathrm{Mu}}=0.5317 \AA
\end{array}
$$

In a solid, the electron wave-function may be distorted owing to the interaction with the electronic structure of the environment. This leads to a change in the hyperfine interaction, which is directly measured by $\mu \mathrm{SR}$. We first discuss the appearance of muonium with an isotropic hyperfine interaction the so called "normal muonium (Mu)" and then a muonium state showing an anisotropic hyperfine coupling constant the "anomalous muonium $\left(\mathrm{Mu}^{*}\right)^{\prime \prime}$.

\section{Normal Muonium}

The Hamiltonian which determines the time evolution of the muon spin in Mu is

$H=A\left|\cdot S-g_{e} \mu_{B}^{e} \mathbf{S} \cdot \mathbf{B}-g_{\mu} \mu_{B}^{\mu}\right| \cdot B$.
In the Zeeman terms $1 / 2 g_{\mathrm{e}} \mu_{B}^{\mathrm{e}}=-9.285$ $\times 10^{-24} \mathrm{~J} / \mathrm{T}$ and $1 / 2 g_{\mu} \mu_{B}^{\mu}=+4.490 \times$ $10^{-26} \mathrm{~J} / \mathrm{T}$ are the magnetic moments of the electron and muon respectively. The hyperfine constant $A$ for the $1 \mathrm{~s}$ state is proportional to $|\psi(0)|^{2}$ the electron density at the muon site:

$A=h v_{0}=-2 / 3 \mu_{\mathrm{o}} g_{\mathrm{e}} \mu_{B}^{\mathrm{e}} g_{\mu} \mu_{B}^{\mu}|\psi(0)|^{2}$. (9) The energy level diagram for $\mathrm{Mu}$ is shown in Fig. 5. In an external transverse field the $\Delta m= \pm 1$ transitions can be observed and from the measured frequencies the hyperfine constant is obtained. Figs. 6-8 show three characteristic results obtained in: the weak field Zeeman region, zero field and the strong field Paschen-Back region. In standard $\mu \mathrm{SR}$, only the intratriplet transitions can be seen because of the limited time resolution (Fig. 6). Our group has made a special effort to improve the resolution to $150 \mathrm{ps}$ (FWHM) and this has allowed us to measure directly the hyperfine frequency $v_{0}$ in zero magnetic field; it also gives greatly improved precision (see Fig. 7). A problem for many years was that in many insulators no signal at all could be detected. The reason was found to be the superhyperfine interaction, the interaction between the $\mathrm{Mu}$ electron and the surrounding nuclear spins which results in a multitude of unresolved small amplitude frequencies. In a high transverse field $(B \approx 1 \mathrm{~T})$, however, where the spins of the muon, electron and nuclei are decoupled, the two lines $v_{12}$ and $v_{34}$ are observable (Fig. 8). The sum of the two lines is exactly equal
Istituto Nazionale di Fisica Nucleare

\section{LABORATORI NAZIONALI DI LEGNARO}

\author{
Two Post-Doctoral Fellowships \\ in Experimental Nuclear Structure Physics
}

Applications are invited for the above posts which are now vacant and are funded by the I.N.F.N. for the period 1 October 1985 30 September 1986. The fellowships are open to non-Italian citizens only. Deadline for applications 1 April 1985. The successful candidates will be expected to initiate and assist with research at the XTU tandem Van de Graaff, which is now operational at the Laboratori Nazionali di Legnaro.

Applicants are expected to hold a Ph.D. degree in nuclear structure physics and should have an aptitude for pursuing research in experimental physics. The salary will be 20.000.000.- lit. gross per annum corresponding to 1.300.000.- net Italian lire per month, plus travel expenses from home institution to Legnaro and return.

Applications with full C.V. and names of two referees and possibly an introduction letter from a senior scientist to:

Prof. Cosimo Signorini, Director,

Laboratori Nazionali di Legnaro - I.N.F.N.

Via Romea, 4 - 35020 Legnaro (Padova) Italy

Tel. : (49) $641200 \quad$ Telex 430384 LNL I 


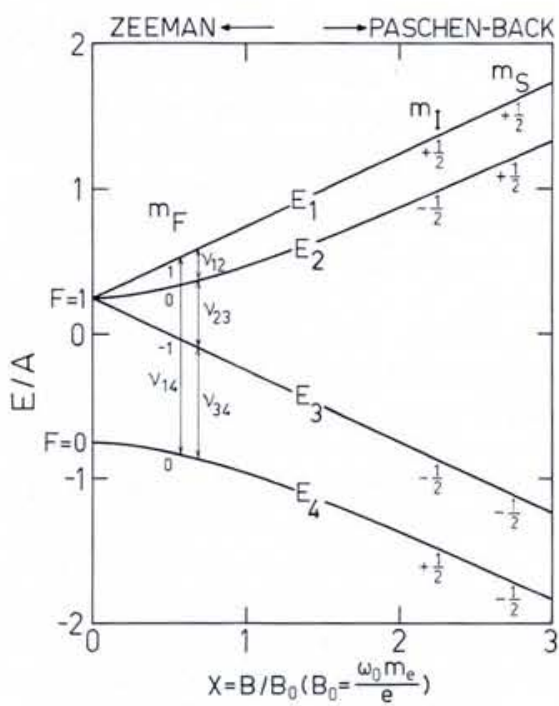

Fig. 5 - Breit-Rabidiagram for normal muonium. The magnetic field $B$ is measured in units $\omega_{o} m_{e} / e=0.15945 \mathrm{~T}$. The $\Delta m= \pm 1$ transitions indicated correspond to the observable $\mu S R$ frequencies in a transverse field.

to the hyperfine frequency $v_{0}$. At the special field $(B=1.13 \mathrm{~T}$ for $\mathrm{Mu}$ in vacuum) the two frequencies $\left(v_{12}=1.923\right.$ $\mathrm{GHz}$ and $v_{34}=2.541 \mathrm{GHz}$ ) have a broad maximum and minimum respectively and thus are insensitive to field inhomogeneities. A few of the measured results are:

$\begin{array}{lr}\mathrm{C} \text { (Diamond) } & v_{\mathrm{o}}(\mathrm{GHz}) \\ \mathrm{Si} & 2.711 \\ \mathrm{Ge} & 2.012 \\ \text { Quartz } & 2.361 \\ \text { Vacuum } & 4.496 \\ 4.463\end{array}$

These hyperfine frequencies show a strong temperature dependence, that is well described by a Debye model. If the hyperfine frequencies are expressed in terms of the Mu $1 \mathrm{~s}$ radius, $a_{\mathrm{Mu}}$ (solid) = $a_{\mathrm{Mu}}$ (vacuum) $\left(A_{\mathrm{vac}} / A_{\text {solid }}\right)^{1 / 3}$, one finds that $\mathrm{Mu}$ and correspondingly a hydrogen atom in diamond, $\mathrm{Si}$, and $\mathrm{Ge}$ is in a state with $a \cong 20 \%$ increased atomic radius. In addition we find strong indications that $\mathrm{Mu}$ (resp. $\mathrm{H}$ ) must be diffusing in $\mathrm{C}$,

Fig. 6 - Spectrum of Si measured in the Zeeman region. Beside the $50.7 \mathrm{MHz}$ cyclotron beam frequency, four lines due to anomalous muonium can be seen.

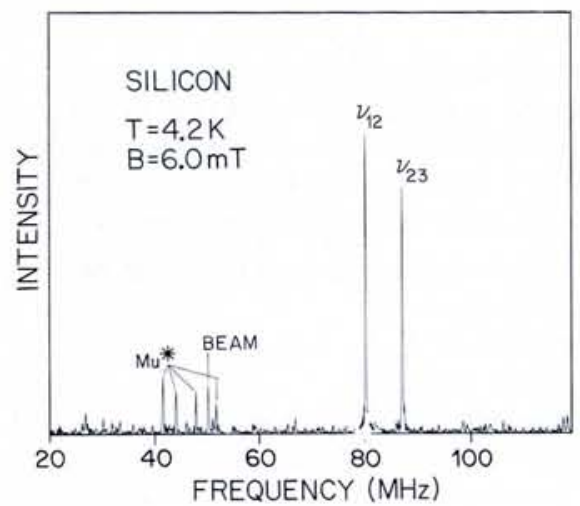

$\mathrm{Si}$, and $\mathrm{Ge}$ down to the lowest temperature measured. So far no consistent explanation for $\mathrm{Mu}(\mathrm{H})$ in semiconductors has been found, although various models have been suggested.

\section{Anomalous Muonium}

In $\mathrm{C}, \mathrm{Si}, \mathrm{Ge}$ and other solids the originally not understood anomalous muonium $\mathrm{Mu}^{*}$ state has been observed. It is described by a Hamiltonian whose hyperfine interaction has axial symmetry about one of the four equivalent $<111\rangle$ directions in the diamond lattice:

$\begin{aligned} H= & A\left(I_{x} S_{x}+I_{y} S_{y}\right)+A_{\| l} I_{z} S_{z} \\ & + \text { Zeeman terms }\end{aligned}$

Fig. 9 shows $\mu$ SR measurement done on diamond powder in zero field. The three frequencies $v_{1}, v_{2}$ and $v_{3}$ observed are:

$$
\begin{aligned}
& v_{1}=\left(A+A_{1}\right) / 2 h \\
& v_{2}=\left(A-A_{1}\right) / 2 h \\
& v_{3}=v_{1}+v_{2}=A / h
\end{aligned}
$$

The expected intensities of the three lines are like $I_{1}: I_{2}: I_{3}=2: 2: 1$. However it is observed that the relative intensities of the three lines are strongly temperature-dependent. The effect is quantitatively explained by a transition of the fast diffusing isotropic $\mathrm{Mu}$ to the localized anisotropic $\mathrm{Mu}^{*}$ state.

Channelling experiments for the positrons emitted from the localized $\mathrm{Mu}^{*}$ in $\mathrm{Si}$ indicate that the $\mathrm{Mu}^{*}$ is localized along a $<111>$ axis at $1.45 \AA$ from the $\mathrm{Si}$ atom in the tetrahedral bond direction relative to a $\mathrm{Si}$ vacancy. $\mathrm{Mu}^{*}$ measurements in external fields depend of course on the crystal orientation and give much additional information on various properties of this interesting light hydrogen isotope.

\section{Other Techniques and Applications}

The $\mu$ SR technique introduced above is called the individual time-differential $\mu \mathrm{SR}$ method in transverse fields since, as indicated in Fig. 1, the decay positrons from individual muons stopped in

Fig. 7 - Spectrum of quartz measured in zero external field. The $4496.2 \pm 0.4 \mathrm{MHz}$ line observed was termed the muonium "heart beat".

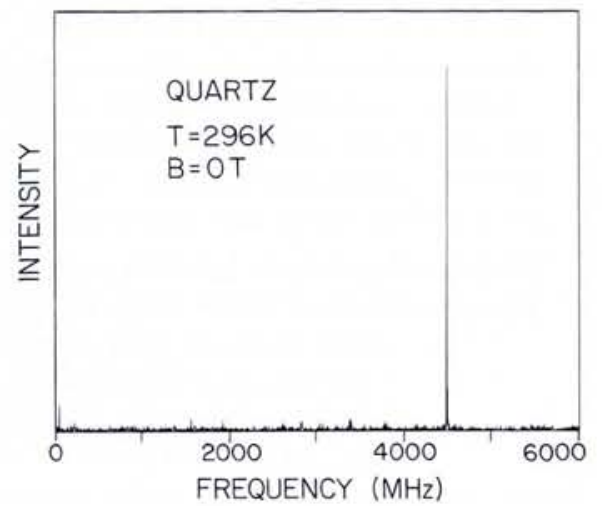

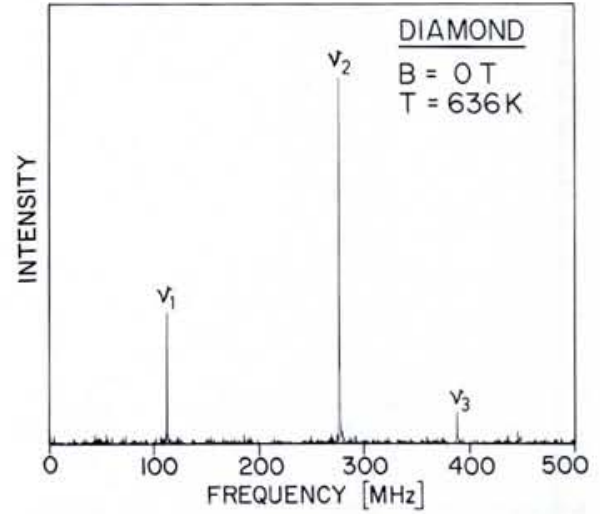

Fig. $9-\mu S R$ frequency spectrum of anomalous muonium in diamond powder measured in zero field.

the sample are recorded one after the other. Other methods which may be favourable to study certain effects involve longitudinal or zero field methods. If pulsed muon beams are available, collective $\mu \mathrm{SR}$ and resonance methods can be used.

These few examples of the $\mu \mathrm{SR}$ method in solid state physics have been selected to give a rough idea of the potential applications of this still new field. Among other possible applications, the use of the muon as a light hydrogen isotope in chemistry, Knight shift experiments, $\mu \mathrm{SR}$ studies in spin glasses, the use of negative muons to probe the nuclear charge distribution, and high precision QED measurements should be mentioned.

\section{REFERENCES}

1. Proceedings of the International Meetings on Muon Spin Rotation Hyperfine Interactions 6 (1979) 1 - 450 .

Ibid 8 (1981) 307 - 834 .

Ibid 17-19 (1984) 1 - 1011.

2. Denison A.B., Graf H., Kündig W. and Meier P.F., Helv. Phys. Acta 52 (1979) 460 517.

3. Muons and Pions in Materials Research, ed. J. Chappert and R.I. Grynszpan (NorthHolland) 1984.

Fig. 8-Muonium in $\mathrm{KCl}$. The hyperfine frequency is $v_{o}=v_{12}+v_{34}$. Owing to the superhyperfine interaction no signal can be seen at low fields.

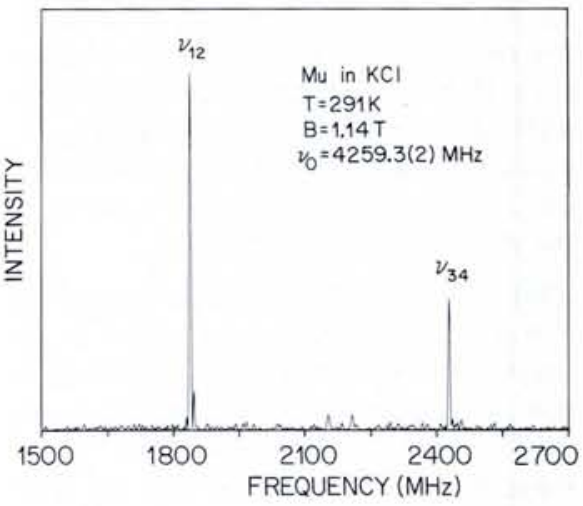

